\title{
The Mittag-Leffler function in the thinning theory for renewal processes
}

\author{
Rudolf GORENFLO ${ }^{(1)}$ and Francesco MAINARDI ${ }^{(2)}$
}

(1) First Mathematical Institute, Free University of Berlin, Arnimallee 3, D-14195 Berlin, Germany.

Deceased (1930-2017)

(2) Department of Physics and Astronomy, University of Bologna, and INFN, Via Irnerio 46, I-40126 Bologna, Italy.

E-mail: francesco.mainardi@bo.infn.it

Keywords: Mittag-Leffler functions, Thinning (Rarefaction), Renewal processes, Queuing theory, Poisson process.

$M S C$ 2000: 26A33, 33E12, 44A10, 60K05. 60K25

\begin{abstract}
The main purpose of this note is to point out the relevance of the Mittag-Leffler probability distribution in the so-called thinning theory for a renewal process with a queue of power law type. This theory, formerly considered by Gnedenko and Kovalenko in 1968 without the explicit reference to the Mittag-Leffler function, was used by the authors in the theory of continuous random walk and consequently of fractional diffusion in a plenary lecture by the late Prof Gorenflo at a Seminar on Anomalous Transport held in Bad-Honnef in July 2006, published in a 2008 book. After recalling the basic theory of renewal processes including the standard and the fractional Poisson processes, here we have revised the original approach by Gnedenko and Kovalenko for convenience of the experts of stochastic processes who are not aware of the relevance of the Mittag-Leffler functions.
\end{abstract}

This note is devoted to the memory of the late Professor Rudolf Gorenflo, passed away on 20 October 2017 at the age of 87. It has been published in the journal Theory of Probability and Mathematical Statistics, Vol. 98, No 1, pp. 100-108 (2018). See http://probability.univ.kiev.ua/tims/ The work of F.M. has been carried out in the framework of the activities of the National Group of Mathematical Physics (GNFM, INdAM). 


\section{Introduction}

In this paper we outline the relevance of the functions of the Mittag-Leffler type in renewal processes and in particular in the thinning theory for longtime behaviour with a generic power law waiting time distribution. In Section 2 we first recall the definition of a generic renewal process with the related probability distribution functions. In Section 3 we discuss the most celebrated renewal process known as the Poisson process defined by an exponential probability density function. Its natural fractional generalization is hence discussed in Section 4 by introducing the so-called renewal process of the Mittag-Leffler type, commonly known as the Fractional Poisson process. Then in Section 5 we consider the thinning theory for a renewal process with a queue of power law type, thereby leaning the presentation of Gnedenko and Kovalenko in 1968 pointing out the key role of the Mittag-Leffler function. Finally, conclusions are drown in Section 6.

\section{Essentials of renewal theory}

The concept of renewal process has been developed as a stochastic model for describing the class of counting processes for which the times between successive events are independent identically distributed (iid) non-negative random variables, obeying a given probability law. These times are referred to as waiting times or inter-arrival times. For more details see e.g. the classical treatises by Khintchine [16, Cox [6], Gnedenko \& Kovalenko [11, Feller [9], and the most recent books by Ross [24, by Beichelt [2], and by Mitov and Omey [20]. jut to cite the treatises that have mostly attracted our attention.

For a renewal process having waiting times $T_{1}, T_{2}, \ldots$, let

$$
t_{0}=0, \quad t_{k}=\sum_{j=1}^{k} T_{j}, \quad k \geq 1 .
$$

That is $t_{1}=T_{1}$ is the time of the first renewal, $t_{2}=T_{1}+T_{2}$ is the time of the second renewal and so on. In general $t_{k}$ denotes the $k$ th renewal.

The process is specified if we know the probability law for the waiting times. In this respect we introduce the probability density function ( $p d f)$ $\phi(t)$ and the (cumulative) distribution function $\Phi(t)$ so defined:

$$
\phi(t):=\frac{d}{d t} \Phi(t), \quad \Phi(t):=P(T \leq t)=\int_{0}^{t} \phi\left(t^{\prime}\right) d t^{\prime} .
$$


When the non-negative random variable represents the lifetime of technical systems, it is common to refer to $\Phi(t)$ as to the failure probability and to

$$
\Psi(t):=P(T>t)=\int_{t}^{\infty} \phi\left(t^{\prime}\right) d t^{\prime}=1-\Phi(t),
$$

as to the survival probability, because $\Phi(t)$ and $\Psi(t)$ are the respective probabilities that the system does or does not fail in $(0, T]$. A relevant quantity is the counting function $N(t)$ that indeed defines the renewal process as

$$
N(t):=\max \left\{k \mid t_{k} \leq t, k=0,1,2, \ldots\right\},
$$

that represents the effective number of events before or at instant $t$. In particular we have $\Psi(t)=P(N(t)=0)$. Continuing in the general theory we set $F_{1}(t)=\Phi(t), f_{1}(t)=\phi(t)$, and in general

$$
F_{k}(t):=P\left(t_{k}=T_{1}+\ldots+T_{k} \leq t\right), f_{k}(t)=\frac{d}{d t} F_{k}(t), k \geq 1,
$$

thus $F_{k}(t)$ represents the probability that the sum of the first $k$ waiting times is less or equal $t$ and $f_{k}(t)$ its density. Then, for any fixed $k \geq 1$ the normalization condition for $F_{k}(t)$ is fulfilled because

$$
\lim _{t \rightarrow \infty} F_{k}(t)=P\left(t_{k}=T_{1}+\ldots+T_{k}<\infty\right)=1 .
$$

In fact, the sum of $k$ random variables each of which is finite with probability 1 is finite with probability 1 itself. By setting for consistency $F_{0}(t) \equiv 1$ and $f_{0}(t)=\delta(t)$, the Dirac delta function in the sense of Gel'fand and Shilov [10] 1 , we also note that for $k \geq 0$ we have

$$
P(N(t)=k):=P\left(t_{k} \leq t, t_{k+1}>t\right)=\int_{0}^{t} f_{k}\left(t^{\prime}\right) \Psi\left(t-t^{\prime}\right) d t^{\prime}
$$

We now find it convenient to introduce the simplified $*$ notation for the convolution between two causal well-behaved (generalized) functions $f(t)$ and $g(t)$

$$
\int_{0}^{t} f\left(t^{\prime}\right) g\left(t-t^{\prime}\right) d t^{\prime}=(f * g)(t)=(g * f)(t)=\int_{0}^{t} f\left(t-t^{\prime}\right) g\left(t^{\prime}\right) d t^{\prime} .
$$

\footnotetext{
${ }^{1}$ We find it convenient to recall the formal representation of this generalized function in $\mathbf{R}^{+}$,

$$
\delta(t):=\frac{t^{-1}}{\Gamma(0)}, \quad t \geq 0 .
$$
}


Being $f_{k}(t)$ the $p d f$ of the sum of the $k$ iid random variables $T_{1}, \ldots, T_{k}$ with $p d f \phi(t)$, we easily recognize that $f_{k}(t)$ turns out to be the $k$-fold convolution of $\phi(t)$ with itself,

$$
f_{k}(t)=\left(\phi^{* k}\right)(t)
$$

so Eq. (2.7) simply reads:

$$
P(N(t)=k)=\left(\phi^{* k} * \Psi\right)(t) .
$$

Because of the presence of convolutions a renewal process is suited for the Laplace transform method. Throughout this paper we will denote by $\widetilde{f}(s)$ the Laplace transform of a sufficiently well-behaved (generalized) function $f(t)$ according to

$$
\mathcal{L}\{f(t) ; s\}=\widetilde{f}(s)=\int_{0}^{+\infty} \mathrm{e}^{-s t} f(t) d t, \quad s>s_{0},
$$

and for $\delta(t)$ consistently we will have $\widetilde{\delta}(s) \equiv 1$. Note that for our purposes we agree to take $s$ real. We recognize that (2.9) reads in the Laplace domain

$$
\mathcal{L}\{P(N(t)=k) ; s\}=[\widetilde{\phi}(s)]^{k} \widetilde{\Psi}(s),
$$

where, using (2.3),

$$
\widetilde{\Psi}(s)=\frac{1-\widetilde{\phi}(s)}{s}
$$

\section{The Poisson process as a renewal process}

The most celebrated renewal process is the Poisson process characterized by a waiting time $p d f$ of exponential type,

$$
\phi(t)=\lambda \mathrm{e}^{-\lambda t}, \quad \lambda>0, \quad t \geq 0 .
$$

The process has no memory being a Lévy process. The moments of waiting times of order $1,2, \ldots \mathrm{n}$ turn out to be

$$
\langle T\rangle=\frac{1}{\lambda}, \quad\left\langle T^{2}\right\rangle=\frac{1}{\lambda^{2}}, \quad \ldots, \quad\left\langle T^{n}\right\rangle=\frac{1}{\lambda^{n}}, \ldots,
$$

and the survival probability is

$$
\Psi(t):=P(T>t)=\mathrm{e}^{-\lambda t}, \quad t \geq 0 .
$$


We know that the probability that $k$ events occur in the interval of length $t$ is

$$
P(N(t)=k)=\frac{(\lambda t)^{k}}{k !} \mathrm{e}^{-\lambda t}, \quad t \geq 0, \quad k=0,1,2, \ldots
$$

The probability distribution related to the sum of $k$ iid exponential random variables is known to be the so-called Erlang distribution (of order $k$ ). The corresponding density (the Erlang pdf) is thus

$$
f_{k}(t)=\lambda \frac{(\lambda t)^{k-1}}{(k-1) !} \mathrm{e}^{-\lambda t}, \quad t \geq 0, \quad k=1,2, \ldots,
$$

so that the Erlang distribution function of order $k$ turns out to be

$$
F_{k}(t)=\int_{0}^{t} f_{k}\left(t^{\prime}\right) d t^{\prime}=1-\sum_{n=0}^{k-1} \frac{(\lambda t)^{n}}{n !} \mathrm{e}^{-\lambda t}=\sum_{n=k}^{\infty} \frac{(\lambda t)^{n}}{n !} \mathrm{e}^{-\lambda t}, \quad t \geq 0 .
$$

In the limiting case $k=0$ we recover $f_{0}(t)=\delta(t), F_{0}(t) \equiv 1, t \geq 0$.

The formulas (3.4)-(3.6) can easily obtained by using the technique of the Laplace transform sketched in the previous section noting that for the Poisson process we have:

$$
\widetilde{\phi}(s)=\frac{\lambda}{\lambda+s}, \quad \widetilde{\Psi}(s)=\frac{1}{\lambda+s},
$$

and for the Erlang distribution:

$$
\widetilde{f}_{k}(s)=[\widetilde{\phi}(s)]^{k}=\frac{\lambda^{k}}{(\lambda+s)^{k}}, \quad \widetilde{F}_{k}(s)=\frac{[\widetilde{\phi}(s)]^{k}}{s}=\frac{\lambda^{k}}{s(\lambda+s)^{k}} .
$$

We also recall that the survival probability for the Poisson renewal process obeys the ordinary differential equation (of relaxation type)

$$
\frac{d}{d t} \Psi(t)=-\lambda \Psi(t), \quad t \geq 0 ; \quad \Psi\left(0^{+}\right)=1
$$

\section{The renewal process of the Mittag-Leffler type}

A "fractional" generalization of the Poisson renewal process is simply obtained by generalizing the differential equation (3.9) replacing there the first derivative with the integro-differential operator ${ }_{t} D_{*}^{\beta}$ that is interpreted as the fractional derivative of order $\beta$ in Caputo's sense. 
For a sufficiently well-behaved function $f(t)(t \geq 0)$ we define the Caputo time fractional derivative of order $\beta$ with $0<\beta<1$ through

$$
\mathcal{L}\left\{{ }_{t} D_{*}^{\beta} f(t) ; s\right\}=s^{\beta} \widetilde{f}(s)-s^{\beta-1} f\left(0^{+}\right), \quad f\left(0^{+}\right):=\lim _{t \rightarrow 0^{+}} f(t),
$$

so that

$$
{ }_{t} D_{*}^{\beta} f(t):=\frac{1}{\Gamma(1-\beta)} \int_{0}^{t} \frac{f^{\prime}(\tau)}{(t-\tau)^{\beta}} d \tau, \quad 0<\beta<1 .
$$

Such operator has been referred to as the Caputo fractional derivative since it was introduced by Caputo in the late 1960's for modelling the energy dissipation in the rheology of the Earth, see [3, 4]. Soon later this derivative was adopted by Caputo and Mainardi in the framework of the linear theory of viscoelasticity, see [5].

The reader should observe that the Caputo fractional derivative differs from the usual Riemann-Liouville (R-L) fractional derivative

$$
{ }_{t} D^{\beta} f(t):=\frac{d}{d t}\left[\frac{1}{\Gamma(1-\beta)} \int_{0}^{t} \frac{f(\tau) d \tau}{(t-\tau)^{\beta}}\right], \quad 0<\beta<1 .
$$

Following the approach by Mainardi et al. [19], we write, taking for simplicity $\lambda=1$,

$$
{ }_{t} D_{*}^{\beta} \Psi(t)=-\Psi(t), \quad t>0, \quad 0<\beta \leq 1 ; \quad \Psi\left(0^{+}\right)=1 .
$$

We also allow the limiting case $\beta=1$ where all the results of the previous section (with $\lambda=1$ ) are expected to be recovered.

We also allow the limiting case $\beta=1$ where all the results of the previous sub-section (with $\lambda=1$ ) are expected to be recovered. In fact, taking $\lambda=1$ simply means a normalized way of scaling the variable $t$.

For our purpose we need to recall the Mittag-Leffler function as the natural "fractional" generalization of the exponential function, that characterizes the Poisson process. The Mittag-Leffler function of parameter $\beta$ is defined in the complex plane by the power series

$$
E_{\beta}(z):=\sum_{n=0}^{\infty} \frac{z^{n}}{\Gamma(\beta n+1)}, \quad \beta>0, \quad z \in \mathbf{C} .
$$

It turns out to be an entire function of order $\beta$ which reduces for $\beta=1$ to $\exp (z)$. For detailed information on the Mittag-Leffler-type functions and 
their Laplace transforms the reader may consult e.g. [8, 13, 22] and the most recent monograph by Gorenflo et al. [12].

The solution of Eq. (4.4) is known to be, see e.g. [5, 18]

$$
\Psi(t)=E_{\beta}\left(-t^{\beta}\right), \quad t \geq 0, \quad 0<\beta \leq 1,
$$

so

$$
\phi(t):=-\frac{d}{d t} \Psi(t)=-\frac{d}{d t} E_{\beta}\left(-t^{\beta}\right), \quad t \geq 0, \quad 0<\beta \leq 1 .
$$

Then, the corresponding Laplace transforms read

$$
\widetilde{\Psi}(s)=\frac{s^{\beta-1}}{1+s^{\beta}}, \quad \widetilde{\phi}(s)=\frac{1}{1+s^{\beta}}, \quad 0<\beta \leq 1 .
$$

Hereafter, we find it convenient to summarize the most relevant features of the functions $\Psi(t)$ and $\phi(t)$ when $0<\beta<1$. We begin to quote their series expansions for $t \rightarrow 0^{+}$and asymptotics for $t \rightarrow \infty$,

$$
\Psi(t)=\sum_{n=0}^{\infty}(-1)^{n} \frac{t^{\beta n}}{\Gamma(\beta n+1)} \sim \frac{\sin (\beta \pi)}{\pi} \frac{\Gamma(\beta)}{t^{\beta}}, \quad t \rightarrow \infty, \quad 0<\beta<1,
$$

and

$\phi(t)=\frac{1}{t^{1-\beta}} \sum_{n=0}^{\infty}(-1)^{n} \frac{t^{\beta n}}{\Gamma(\beta n+\beta)} \sim \frac{\sin (\beta \pi)}{\pi} \frac{\Gamma(\beta+1)}{t^{\beta+1}}, \quad t \rightarrow \infty, \quad 0<\beta<1$.

In contrast to the Poissonian case $\beta=1$, in the case $0<\beta<1$ for large $t$ the functions $\Psi(t)$ and $\phi(t)$ no longer decay exponentially but algebraically. As a consequence of the power-law asymptotics the fractional Poisson process for $\beta<1$ turns be no longer Markovian as for $\beta=1$ but of long-memory type. However, we recognize that for $0<\beta<1$ both functions $\Psi(t), \phi(t)$ keep the "completely monotonic" character of the Poissonian case. Complete monotonicity of the functions $\Psi(t)$ and $\phi(t)$ means

$$
(-1)^{n} \frac{d^{n}}{d t^{n}} \Psi(t) \geq 0, \quad(-1)^{n} \frac{d^{n}}{d t^{n}} \phi(t) \geq 0, \quad n=0,1,2, \ldots, \quad t \geq 0,
$$

or equivalently, their representability as real Laplace transforms of nonnegative generalized functions (or measures), see e.g. [9].

For the generalizations of Eqs (3.4) and (3.5)-(3.6), characteristic of the Poisson and Erlang distributions respectively, we must point out the Laplace transform

$$
\mathcal{L}\left\{t^{\beta k} E_{\beta}^{(k)}\left(-t^{\beta}\right) ; s\right\}=\frac{k ! s^{\beta-1}}{\left(1+s^{\beta}\right)^{k+1}}, \quad \beta>0, \quad k=0,1,2, \ldots,
$$


with $E_{\beta}^{(k)}(z):=\frac{d^{k}}{d z^{k}} E_{\beta}(z)$, that can be deduced from the book by Podlubny, see (4.80) in [22]. Then, by using the Laplace transforms (4.8) and Eqs (4.6), (4.7), (4.12) in Eqs (2.8) and (2.9), we have the generalized Poisson distribution,

$$
P(N(t)=k)=\frac{t^{k \beta}}{k !} E_{\beta}^{(k)}\left(-t^{\beta}\right), \quad k=0,1,2, \ldots
$$

and the generalized Erlang pdf (of order $k \geq 1$ ),

$$
f_{k}(t)=\beta \frac{t^{k \beta-1}}{(k-1) !} E_{\beta}^{(k)}\left(-t^{\beta}\right)
$$

The generalized Erlang distribution function turns out to be

$$
F_{k}(t)=\int_{0}^{t} f_{k}\left(t^{\prime}\right) d t^{\prime}=1-\sum_{n=0}^{k-1} \frac{t^{n \beta}}{n !} E_{\beta}^{(n)}\left(-t^{\beta}\right)=\sum_{n=k}^{\infty} \frac{t^{n \beta}}{n !} E_{\beta}^{(n)}\left(-t^{\beta}\right) .
$$

For readers' convenience we conclude this section citing other works dealing with the so-called fractional Poisson process from different point of view, see e.g. Laskin [17, Beghin and Orsingher [1].

\section{The Mittag-Leffler distribution as limit for thinned renewal processes}

Now, we provide, in our notation, an outline of the thinning theory for renewal processes essentially following the 1968 approach by Gnedenko and Kovalenko [11. Examples of thinning processes are provided in the the 2006 book by Beichelt [2], where we read For instance, a cosmic particle counter registers only $\alpha$-particles and ignores other types of particles. Or, a reinsurance company is only interested in claims, the size of which exceeds, say, one million dollars.

We must note that other authors, like Szántai [25, 26] speak of rarefaction in place of thinning.

Let us sketch here the essentials of this theory. Denoting by $t_{n}, n=$ $1,2,3, \ldots$ the time instants of events of a renewal process, assuming $0=$ $t_{0}<t_{1}<t_{2}<t_{3}<\ldots$, with i.i.d. waiting times $T_{1}=t_{1}, T_{k}=t_{k}-t_{k-1}$ for $k \geq 2$, (generically denoted by $\mathrm{T}$ ), thinning (or rarefaction) means that for each positive index $k$ a decision is made: the event happening in the instant 
$t_{k}$ is deleted with probability $p$ or it is maintained with probability $q=1-p$, $0<q<1$. This procedure produces a thinned or rarefied renewal process with fewer events (very few events if $q$ is near zero, the case of particular interest) in a moderate span of time.

To compensate for this loss we change the unit of time so that we still have not very few but still a moderate number of events in a moderate span of time. Such change of the unit of time is equivalent to rescaling the waiting time, multiplying it with a positive factor $\tau$ so that we have waiting times $\tau T_{1}, \tau T_{2}, \tau T_{3}, \ldots$, and instants $\tau t_{1}, \tau t_{2}, \tau t_{3}, \ldots$, in the rescaled process.

In other words to bring the distant future into near sight we change the unit of time from 1 to $1 / \tau, 0<\tau \ll 1$.

For the random waiting times $T$ this means replacing $T$ by $\tau T$. Then, having very many events in a moderate span of time we compensate this compression by respeeding the whole process, actually slowing it down so that again we have a moderate number of events in a moderate span of time. Our intention is, vaguely speaking, to dispose on $\tau$ in relation to the rarefaction parameter $q$ in such a way that for $q$ near zero in some sense the "average" number of events per unit of time remains unchanged. In an asymptotic sense we will make these considerations precise.

Denoting by $F(t)=P(T \leq t)$ the probability distribution function of the (original) waiting time $T$, by $f(t)$ its density $(f(t)$ is a generalized function generating a probability measure) so that $F(t)=\int_{0}^{t} f\left(t^{\prime}\right) d t^{\prime}$, and analogously by $F_{k}(t)$ and $f_{k}(\mathrm{t})$ the distribution and density, respectively, of the sum of $k$ waiting times, we have recursively

$$
f_{1}(t)=f(t), \quad f_{k}(t)=\int_{0}^{t} f_{k-1}\left(t-t^{\prime}\right) d F\left(t^{\prime}\right), \text { for } k \geq 2 .
$$

Observing that after a maintained event the next one of the original process is kept with probability $q$ but dropped in favor of the second-next with probability $p q$ and, generally, $n-1$ events are dropped in favor of the $n$ th-next with probability $p^{n-1} q$, we get for the waiting time density of the thinned process the formula

$$
g_{q}(t)=\sum_{n=1}^{\infty} q p^{n-1} f_{n}(t) .
$$

With the modified waiting time $\tau T$ we have

$$
P(\tau T \leq t)=P(T \leq t / \tau)=F(t / \tau)
$$


hence the density $f(t / \tau) / \tau$, and analogously for the density of the sum of $n$ waiting times $f_{n}(t / \tau) / \tau$. The density of the waiting time of the rescaled (and thinned) process now turns out as

$$
g_{q, \tau}(t)=\sum_{n=1}^{\infty} q p^{n-1} f_{n}(t / \tau) / \tau
$$

In the Laplace domain we have $\widetilde{f}_{n}(s)=(\widetilde{f}(s))^{n}$, hence (using $p=1-q$ )

$$
\widetilde{g}_{q}(s)=\sum_{n=1}^{\infty} q p^{n-1}(\widetilde{f}(s))^{n}=\frac{q \widetilde{f}(s)}{1-(1-q) \widetilde{f}(s)},
$$

from which by Laplace inversion we can, in principle, construct the waiting time density of the thinned process. By re-scaling we get

$$
\widetilde{g}_{q, \tau}(s)=\sum_{n=1}^{\infty} q p^{n-1}(\widetilde{f}(\tau s))^{n}=\frac{q \tilde{f}(\tau s)}{1-(1-q) \widetilde{f}(\tau s)} .
$$

Being interested in stronger and stronger thinning (infinite thinning) let us now consider a scale of processes with the parameters $\tau$ (of rescaling) and $q$ (of thinning), with $q$ tending to zero under a scaling relation $q=q(\tau)$ yet to be specified.

We have essentially two cases for the waiting time distribution: its expectation value (the first moment) is finite or infinite. In the first case we put

$$
\mu=\int_{0}^{\infty} t^{\prime} f\left(t^{\prime}\right) d t^{\prime}<\infty, \quad \beta=1 .
$$

In the second case we assume a queue of power law type

$$
\Psi(t):=\int_{t}^{\infty} f\left(t^{\prime}\right) d t^{\prime} \sim \frac{c}{\beta} t^{-\beta}, t \rightarrow \infty, \quad 0<\beta<1 .
$$

Then, by the Tauberian theory (see e.g. [9, 27]) the above conditions mean in the Laplace domain

$$
\widetilde{f}(s)=1-\mu s^{\beta}+o\left(s^{\beta}\right), \quad \text { for } \quad s \rightarrow 0^{+},
$$

with a positive coefficient $\mu$ and $0<\beta \leq 1$. The case $\beta=1$ obviously corresponds to the situation with finite first moment, whereas the case $0<$ $\beta<1$ is related to a power law queue with $c=\mu \Gamma(\beta+1) \sin (\beta \pi) / \pi$. 
Now, passing to the limit of $q \rightarrow 0$ of infinite thinning under the scaling relation

$$
q=\mu \tau^{\beta}, \quad 0<\beta \leq 1,
$$

between the positive parameters $q$ and $\tau$, the Laplace transform of the rescaled density $\widetilde{g_{q, \tau}}(s)$ in $(5.5)$ of the thinned process tends for fixed $s$ to

$$
\widetilde{g}(s)=\frac{1}{1+s^{\beta}},
$$

which corresponds to the Mittag-Leffler density

$$
g(t)=-\frac{d}{d t} E_{\beta}\left(-t^{\beta}\right)=\phi^{M L}(t) .
$$

We note that in the literature the distribution (5.10) is known as positive Linnik distribution, see e.g. [7] and used by Pillai [21] to define the MittagLeffler distribution. Let us remark that Gnedenko and Kovalenko in 1968 obtained (5.10) as the Laplace transform of the limiting density but did not identify it as the Laplace transform of a Mittag-Leffler type function even if this Laplace transform was known since the late 1950's in the Bateman Handbook [8]. Observe once again that in the special case $\beta=1$ we recover as the limiting process the Poisson process, as formerly shown in 1956 by Rényi [23].

\section{Conclusions}

We have revised the basic theory of renewal processes including the standard and fractional Poisson processes. and in particular the thinning theory with a power law queue, in order to out the relevance of the Mittag-Leffler functions. These processes are essential in the theory of continuous time random walks, and, under re-scalng of time and space coordinates, in spacetime fractional diffusion processes, as shown e.g. in papers by Gorenflo and Mainardi, see the survey [15]. For the thinning theory we have followed the original approach based on Laplace transform by Gnedenko and Kovalenko, who, however, had ignored the Mittag-Leffler functions. In practice this

note points out a further application of the functions of the Mittag-Leffler type in stochastic processes, not so well known. 


\section{References}

[1] L. Beghin and E. Orsingher, Iterated elastic Brownian motions and fractional diffusion equations, Stochastic Processes and their Applications 119 (2009), 1975-2003.

[2] F. Beichelt, Stochastic Processes in Science, Engineering and Finance, Chapman \& Hall/CRC', Boca Raton, US. (2006).

[3] M. Caputo, Linear models of dissipation whose $Q$ is almost frequency independent, Part II. Geophys. J. R. Astr. Soc. 13 (1967), 529-539.

[4] M. Caputo, Elasticità e Dissipazione. Bologna, Zanichelli (1969).

[5] M. Caputo and F. Mainardi, Linear models of in anelastic solids. Riv. Nuovo Cimento (Ser. II) 1 (1971), 161-198.

[6] D.R. Cox, Renewal Theory, 2-nd Edn, Methuen, London (1967).

[7] L. Devroye, A note on Linnik's distribution, Statistics 8 Probability Letters 9 (1990), 305-306.

[8] A. Erdélyi, W. Magnus, F. Oberhettinger and F.G. Tricomi, Higher Transcendental Functions, Bateman Project, McGraw-Hill, New York, 1955, Vol 3. [Ch. 18: Miscellaneous Functions, pp. 206-227]

[9] W. Feller, An Introduction to Probability Theory and its Applications, Vol. 2, 2-nd edn. Wiley, New York (1971) .[1-st edn. 1966]

[10] I.M. Gel'fand and G.E. Shilov, Generalized Functions, Vol. 1, Academic Press, New York (1964).

[English translation from the Russian (Nauka, Moscow, 1959)]

[11] B.V Gnedenko and I.N Kovalenko, Introduction to Queueing Theory, Israel Program for Scientific Translations', Jerusalem (1968). (Translated from the Russian)

[12] R. Gorenflo, A. Kilbas, F. Mainardi and S. Rogosin, Mittag-Leffler Functions, Related topics and Applications Spinger, Berlin (2014).

[13] R. Gorenflo and F. Mainardi, Fractional calculus: integral and differential equations of fractional order, in: A. Carpinteri and F. Mainardi (Editors), Fractals and Fractional Calculus in Continuum Mechanics, Springer Verlag, Wien (1997), pp. 223-276. 
[14] R. Gorenflo and F. Mainardi, Anomalous Transport: Foundations and Applications, Ch 4: Continuous time random walk, Mittag-Leffler waiting time and fractional diffusion: mathematical aspects, (R. Klages, G. Radons, I.M. Sololov, eds.), Wiley-VCH, Weinheim, Germany, (2008), pp. 93-127. [E-print: http://arxiv.org/abs/0705.0797

[15] R. Gorenflo and F. Mainardi, Parametric Subordination in Fractional Diffusion Processes, in J. Klafter, S.C. Lim and R. Metzler (Editors), Fractional Dynamics, World Scientific, Singapore (2012), Chapter 10, pp. 229-263 [E-print: http://arxiv.org/abs/1210.8414

[16] A. Ya. Khintchine, Mathematical Methods in the Theory of Queueing, Charles Griffin, London (1960).

[17] N. Laskin, Fractional Poisson processes, Comm. Nonlinear Sci. Num. Sim. 8 (2003), 201-213.

[18] F. Mainardi and R. Gorenflo, Time-fractional derivatives in relaxation processes: a tutorial survey, Fract. Calc. Appl. Anal. 10 (2007), 269-308.

[19] F. Mainardi, R. Gorenflo and E. Scalas, A fractional generalization of the Poisson processes Vietnam Journal of Mathematics 32 SI (2004), $53-64$.

[20] K.V. Mitov and E. Omey, Renewal Processes, Springer, Heidelberg (2014).

[21] R.N. Pillai, On Mittag-Leffler functions and related distributions, Ann. Inst. Statist. Math. 42 No 1 (1990), 157-161.

[22] I. Podlubny, Fractional Differential Equations, Academic Press, New York (1999).

[23] A. Renyi, A characteristic of the Poisson stream, Proc. Math. Inst. Hungarica Acad. Sci. 1 (1956), no. 4, 563-570. [In Hungarian]

[24] S.M. Ross, Introduction to Probability Models, 6-th Edn, Academic Press, New York (1997).

[25] T. Szàntai, Limiting distribution for the sums of random number of random variables concerning the rarefaction of recurrent events, Studia Scientiarum Mathematicarum Hungarica 6 (1971), 443-452. 
[26] T. Szàntai, On an invariance problem related to different rarefactions of recurrent event, Studia Scientiarum Mathematicarum Hungarica 6 (1971), 453-456.

[27] D.V. Widder, The Laplace Transform, Princeton University Press, Princeton (1946). 